\section{Contribución a la seguridad alimentaria en el municipio de Cabaiguán.}

\section{Contribution to the food safety on Cabaiguan municipality.}

\author{
Onelia Guevara Reyes ${ }^{1}$, Francisco Hernández \\ Somoza, Raquel de la Cruz Soriano y \\ Nelson León Orellana \\ ${ }^{1}$ Universidad de Sancti Spíritus, Cuba \\ onelia@uniss.edu.cu
} Recibido: $17 / 06 / 2019$ Aceptado: $20 / 11 / 2019$

Publicado: $27 / 12 / 2019$

\title{
RESUMEN
}

La innovación agropecuaria local es clave en el desarrollo de niveles productivos en las producciones agropecuarias para el logro de la seguridad alimentaria de cada territorio. El objetivo de la ponencia consistió en analizar los resultados de la aplicación de un sistema de acciones como contribución a la seguridad alimentaria en Cabaiguán. Se utilizó la metodología que sustenta la ejecución del Proyecto de Innovación Agropecuaria Local y el procedimiento para la capacitación agropecuaria, mediante el aprendizaje en la acción. Este proceso se realizó en los grupos de aprendizaje, donde cada grupo planificó, implementó las actividades; reflexionó sobre las lecciones aprendidas y re-planificó para un próximo ciclo de aprendizaje. Se comenzó con la selección y designación de las entidades productivas vinculadas directamente al proyecto, de productores de confianza, quienes, de conjunto con el gobierno, la Dirección Municipal de la Agricultura, y el Centro Universitario Municipal constituyeron una plataforma local multiactoral de gestión para la innovación agropecuaria. La experimentación campesina logró impactos importantes relacionados con una mayor eficiencia en la finca, medida en el aumento del rendimiento de sus parcelas y de la diversidad por el uso mayor de especies vegetales y animales. Se iniciaron los Grupos de Innovación Agropecuaria Local, como espacios de participación con enfoque de género, la impartición de acciones de capacitación para la implementación de buenas prácticas aportadas por el sistema de innovación local, el intercambio con productores de experiencia y la socialización de resultados por parte de investigadores, docentes, estudiantes y productores en un evento regional.

PALABRAS CLAVE: capacitación, innovación agropecuaria, seguridad alimentaria.

\section{ABSTRACT}

The objective of this work was to analyze the result of the application of an action system as a contribution to the food safety in Cabaiguan. The methodology used was the one that sustain the realization of the Local Agricultural Innovation Project and the Agricultural capacitation, through the learning action. This process has been done on the learning group, where each group planned the action and activities, implemented, analyze based on results and re-planned for a new learning cycle. We start with the selection and designation of the productive entities bound to the project, truthful producers; they along with the government, Agricultural Municipality Management and Municipality University Center were a local multi actor platform for agricultural innovation. The farmers' experimentation achieved great impact related with higher farm efficiency; it was measure on the production rise and diversity of vegetables and animals. Local Agricultural Innovation Groups (GIAL) have been initiated, as participation spaces with a gender view, the capacitation for the implementation of good practices delivered for the local innovation system, the interchange with 
experienced producers and the socialization of the results by the investigators, scholars, students and producers in a regional event.

KEYWORDS: agricultural innovation, capacitation, food safety.

\section{INTRODUCCIÓN}

Los conocimientos y saberes acumulados requieren de su introducción en la práctica agropecuaria, especialmente en el contexto actual donde la producción de alimentos ha sido declarada por el Estado cubano como un elemento clave de seguridad nacional, en una etapa marcada por un fuerte debate sobre:

Los desafíos actuales de la agricultura donde se analizan alternativas para el uso de formas descentralizadas y participativas de innovar, producir y comercializar alimentos.

En Cuba existen resultados científicos en las diferentes instituciones en el sector agropecuario y se implementan diferentes proyectos. (Tejeda, 2015, p. 5)

La capacitación y aprendizaje por parte de los productores, independientemente de las formas de gestión, contribuyen a que las buenas prácticas gestionadas por el sistema de innovación local sean implementadas por las estrategias municipales y los grupos de innovación agropecuaria locales. Por tanto, es fundamental la creación y sistematización de espacios de participación de los implicados (cuadros, facilitadores y productores) con el objetivo de consolidar la innovación agropecuaria local sustentada en los procesos y en las dinámicas participativas. Es clave en el estado de desarrollo social y correspondencia con el modelo económico actual «favorecer la participación de las mujeres y los jóvenes de comunidades rurales participen y se beneficien prioritariamente» (Romero et al., 2009, p. 10).

Por lo antes expuesto resulta esencial el logro de la extensión agraria y el desarrollo rural. En este sentido se asume el concepto de la extensión como «la mejora de la gestión del conocimiento agrario o como la intervención sistemática sobre los procesos de generación, intercambio y utilización de conocimiento e información agraria» (Sánchez de Puerta, 1996, p. 3).

La agricultura es «uno de los sectores claves en los que se han realizado importantes trasformaciones orientadas a hacer frente a la imperiosa necesidad de incrementar la producción de alimentos y reducir el nivel de importaciones» (Hernández et al., 2016, p. 9). Existen varios factores que determinan la necesidad de impulsar la innovación agropecuaria local, entre los que se pueden mencionar el déficit de insumos para el sector agrario existente, la producción de alimentos en condiciones de estrés biótico y abiótico, la producción agropecuaria se realiza en condiciones ambientales muy específicas. Por tanto, se hace cada vez más difícil generalizar una variedad o paquete tecnológico en extensas y diferentes áreas geográficas, lo que hace que los productos del sistema formal de innovación tengan niveles de adopción y diseminación menores que lo esperado.

Estas necesidades tienen sus particularidades en cada municipio del país; en el caso particular de Cabaiguán, municipio cubano perteneciente a la provincia Sancti Spíritus, se ejecuta el Proyecto de Innovación Agropecuaria Local (PIAL), en un contexto enfocado en el diseño e implementación de diversas medidas orientadas a la actualización del modelo económico cubano y que garanticen la seguridad alimentaria según se expresa en los Lineamientos de la Política Económica y Social del Partido y la Revolución (2016). En este documento se le ha dado especial atención al monitoreo y evaluación participativa y a la difusión de las experiencias sistematizadas. Igualmente se han tratado los cuatro temas transversales: Diversidad genética y tecnológica; transversalización de género; 
adaptación y mitigación al cambio climático; y trabajo con jóvenes. El objetivo del presente trabajo consiste en analizar los resultados de la aplicación de un sistema de acciones como contribución a la seguridad alimentaria en Cabaiguán.

\section{MATERIALES Y MÉTODOS}

Las decisiones tomadas entre otras fueron: revisar las acciones, ver las limitaciones de la planeación, trazar nuevas metas e iniciativas, trabajar arduamente, con más claridad en función de darle continuidad a la interminable tarea de la materialización de la innovación agropecuaria local. Se utilizó la metodología o marco lógico que sustenta la ejecución del Proyecto de Innovación Agropecuaria Local (PIAL) (Instituto Nacional de Ciencias Agrícolas [INCA], 2012) y el procedimiento para la capacitación agropecuaria resumido por Marzin, López y Cid (2003) y Ramírez, Hernández y Marrero (2009).

También fue empleado el Aprendizaje e Investigación en la Acción (AIA) conformado por una serie de metodologías que contribuyen a construir alternativas que mejoran las relaciones entre los actores locales, basado en una participación activa de los mismos. Se propuso que este proceso de AIA se desarrollara en los grupos de aprendizaje, donde cada grupo planificó las acciones o actividades (plan operativo de acción o propuesta de intervención), las implementó (ejecución de las acciones), reflexionó sobre las lecciones aprendidas (identificación de buenas y malas prácticas) y re-planificó (nuevo plan operativo de acción) para un próximo ciclo de aprendizaje.

Dicha metodología se aplicó en cada Grupo de Innovación Agropecuaria Local (GIAL), las Cooperativas de Producción Agropecuaria (CPA) y las Cooperativas de Créditos y Servicios (CCS) incorporadas al proyecto a través del procedimiento siguiente:

Establecer la jerarquía de objetivos; implementar las acciones de Innovación Agropecuaria Local; realizar la medición del impacto: se requiere establecer indicadores de impacto, las fuentes de datos y los supuestos; y evaluar efectos: se requiere establecer indicadores de efecto, las fuentes de datos, los supuestos y los productos e indicadores de producto. (Instituto Nacional de Ciencias Agrícolas, 2012, p. 12)

El desarrollo de la investigación se sustentó de forma general en el método dialéctico como método universal del conocimiento, permitiendo determinar en el objeto de estudio las contradicciones, los nexos y las soluciones al problema investigado. Además, se realizó la caracterización y diagnóstico del municipio en la esfera agropecuaria. Se aplicaron métodos empíricos como encuestas y entrevistas a directivos, técnicos, productores para la selección de las muestras de confianza, la revisión de documentos, la observación y como técnicas la guía de observación, la guía de revisión de documentos y la encuesta. Para la propuesta de las acciones se asumieron determinados rasgos generales (De Armas, Lorences y Perdomo, 2003), según estos autores a dichas acciones las distinguen el enfoque sistémico, en el que predominan las relaciones de coordinación estructuradas por etapas relacionadas con las acciones de orientación, ejecución y control. Estas acciones se caracterizaron por ser dialécticas, contextuales, personalizadas, dinámicas, objetivas, operativas, formativas y sistemáticas.

\section{RESULTADOS Y DISCUSIÓN}

De manera oficial el municipio Cabaiguán se insertó en el año 2016, aunque ya se habían ejecutado acciones previas desde el año 2012 a solicitud de la Universidad de Sancti Spíritus José Martí (UNISS), fundamentalmente en actividades de capacitación y prueba de variedades. En la tabla 1 se 
presentan los diversos componentes estructurales para su ejecución, descripción y los roles desempeñados por cada uno de ellos.

Tabla 1. Caracterización de los componentes estructurales del proyecto.

\begin{tabular}{|c|c|c|}
\hline $\begin{array}{l}\text { Componentes del } \\
\text { proyecto }\end{array}$ & $\begin{array}{l}\text { Composición/ } \\
\text { Descripción }\end{array}$ & Roles fundamentales \\
\hline $\begin{array}{l}\text { Plataformas Locales para la } \\
\text { Innovación Agropecuaria }\end{array}$ & $\begin{array}{l}\text { Formada por } \\
\text { representantes del } \\
\text { Gobierno Municipal, los } \\
\text { GIAL, representantes de } \\
\text { Asociación Nacional de } \\
\text { Agricultores Pequeños } \\
\text { (ANAP), Asociación Cubana } \\
\text { de Producción Animal } \\
\text { (ACPA), Asociación Cubana } \\
\text { de Técnicos Agrícolas y } \\
\text { Forestales (ACTAF), } \\
\text { Federación de Mujeres } \\
\text { Cubanas (FMC), Ministerio } \\
\text { de educación Superior } \\
\text { (MES), Ministerio de la } \\
\text { Agricultura (MINAG), } \\
\text { Ministerio de Ciencia } \\
\text { Tecnología y Medio } \\
\text { Ambiente(CITMA), } \\
\text { Ministerio de Comercio } \\
\text { Exterior (MINCEX). }\end{array}$ & $\begin{array}{l}\text { - Funcionan como espacios locales de } \\
\text { interacción, intercambio y } \\
\text { armonización a nivel municipal para } \\
\text { diseminar en las instancias y actores } \\
\text { claves del municipio y la provincia, las } \\
\text { buenas prácticas del PIAL (el } \\
\text { aprendizaje en la acción, el acceso a la } \\
\text { diversidad y otras), así como los } \\
\text { resultados de monitoreo y evaluación. } \\
\text { - Planificación estratégica de la } \\
\text { implementación del Proyecto en los } \\
\text { municipios, de acuerdo a las } \\
\text { especificidades y prioridades del } \\
\text { territorio, que tributen a los objetivos y } \\
\text { principios del mismo. } \\
\text { - Identifican iniciativas y programas } \\
\text { claves en el ámbito agropecuario a } \\
\text { nivel municipal y diseñan e } \\
\text { implementan acciones de armonización } \\
\text { y sinergias. } \\
\text { - Buscan y socializan nuevas } \\
\text { oportunidades de fondos. }\end{array}$ \\
\hline $\begin{array}{l}\text { Grupos de Innovación } \\
\text { Agropecuaria Local (GIAL) }\end{array}$ & $\begin{array}{l}\text { Formados por } \\
\text { agricultoras/es de varias } \\
\text { fincas y/o cooperativas y } \\
\text { otras formas } \\
\text { agroproductivas, y en } \\
\text { ocasiones por otros actores } \\
\text { locales. }\end{array}$ & $\begin{array}{l}\text { - Introducción, experimentación, } \\
\text { discusión y diseminación local de } \\
\text { nuevas variantes agroproductivas, } \\
\text { tecnológicas y/o de comercialización, } \\
\text { que contribuyan a la seguridad y } \\
\text { soberanía alimentaria, y adaptación al } \\
\text { cambio climático de sus comunidades. } \\
\text { - Diversificación de sus producciones } \\
\text { con carácter agroecológico, con énfasis } \\
\text { en incrementar el valor agregado y la } \\
\text { calidad de las producciones, } \\
\text { encaminado a la posible diferenciación } \\
\text { de los productos de la innovación en } \\
\text { los espacios locales de mercado. } \\
\text { - Contribución y participación en los } \\
\text { procesos de aprendizaje en la acción. }\end{array}$ \\
\hline
\end{tabular}


Muestras de confianza
- Diseminan las buenas prácticas incorporadas a sus producciones entre otros actores del contexto local, nacional e internacional.

- Tenencia de una finca diversificada, con eficiencia en indicadores económicos y productivos.

- Se logra la inserción de jóvenes y mujeres en las diferentes labores.

- Logro de resultados destacados en el movimiento agroecológico.

- Introducción de tecnologías avanzadas en la producción agropecuaria.

- Uso eficiente y racional del agua.

- Socialización de los resultados en los GIAL a través de diferentes formas de capacitación y la participación en eventos.

Fuente: Elaboración propia.

El aporte principal de la innovación presentada consistió en la ejecución del procedimiento que se describe en las acciones científico-tecnológicas. Con la metodología de AIA se logró la construcción de un nuevo conocimiento por diferentes actores locales a partir de los problemas identificados, así como las soluciones propuestas e implementadas (tabla 2).

Tabla 2. Grupos de Aprendizaje y productos.

\begin{tabular}{|c|c|c|}
\hline $\begin{array}{l}\text { Grupo de } \\
\text { aprendizaje }\end{array}$ & Composición & Objetivos \\
\hline $\begin{array}{l}\text { Dinamizadores de la } \\
\text { innovación local }\end{array}$ & $\begin{array}{l}\text { Instituciones rectoras provinciales del } \\
\text { PIAL: UNISS y el Centro Universitario } \\
\text { Municipal Capitán Silverio Blanco } \\
\text { Núñez (CUM). }\end{array}$ & $\begin{array}{l}\text { Organizar los grupos de } \\
\text { aprendizaje que se identifican en } \\
\text { esta tabla. }\end{array}$ \\
\hline $\begin{array}{l}\text { Grupos de } \\
\text { innovación local }\end{array}$ & $\begin{array}{l}\text { Actores claves de la cadena } \\
\text { agroalimentaria. }\end{array}$ & $\begin{array}{l}\text { Facilitar el acceso y la } \\
\text { disponibilidad de mayor } \\
\text { diversidad de productos } \\
\text { agropecuarios a los } \\
\text { consumidores. }\end{array}$ \\
\hline $\begin{array}{l}\text { Técnicos- } \\
\text { Universitarios }\end{array}$ & $\begin{array}{l}\text { Profesionales que trabajan asociados } \\
\text { a la ACTAF y la ACPA en la provincia y }\end{array}$ & $\begin{array}{l}\text { Ofrecer servicios técnicos en } \\
\text { todos los eslabones de la cadena }\end{array}$ \\
\hline
\end{tabular}


el municipio.

Plataformas Locales de Desarrollo Agropecuario

Monitoreo y

Evaluación

Administración y

Logística
Decisores de políticas interesados en el PIAL y miembros destacados de las cadenas agroalimentarias locales (Gobierno, CUM, ANAP, Empresas, ACTAF, Brigadas Técnico Juveniles (BTJ), Unión de Jóvenes Comunistas (UJC), FMC, Agricultura Urbana, CITMA, GIAL.

Representantes de los sectores claves relacionados con el sector agropecuario.

Administradores, contadores y logísticos de PIAL. agroalimentaria.

Facilitar el mejoramiento del desempeño de las cadenas agroalimentarias en las zonas o escenarios de aprendizaje seleccionados.

Diseñar un sistema de monitoreo y evaluación participativo.

Facilitar una gestión descentralizada, que permita a las Plataformas Locales para la Innovación Agropecuaria municipales una mayor participación en la planificación estratégica y financiera y la ejecución financiera transparente de los fondos del mismo.

Fuente: Elaboración propia.

Como resultado se planificaron e implementaron las acciones siguientes:

1. Seleccionar cuatro entidades productivas vinculadas al PIAL en el municipio Cabaiguán: CPA Aramís Pérez, CPA 10 de octubre, CPA Cuba Nueva, CCS Sergio Soto.

2. Seleccionar seis productores de confianza (productores destacados en los diferentes renglones productivos de la localidad, con prioridad en jóvenes y mujeres).

3. Establecer ocho Grupos de Innovación Agropecuaria Local (GIAL) que abarcan las principales actividades sociales, económicas y productivas: tabacaleros, plantas ornamentales, oleaginosas, de granos, de semillas, artesanos, cría porcina y minindustria.

4. Establecer la plataforma local multiactoral liderada por el vicepresidente del Gobierno municipal que atiende la Esfera agroalimentaria y que vincula a los principales factores del territorio: CITMA, ANAP, ACTAF, UJC, FMC, BTJ, CUM, Enseñanza Técnico Profesional del Ministerio de Educación (MINED), Partido Comunista de Cuba (PCC), CPA y CCS implicados, responsables de los GIAL, Agricultura Urbana, Empresa Agropecuaria, líderes de otros proyectos (BIOMAS, HÁBITAT y AGROCADENAS), Comunales, Delegación de la Agricultura, Comercio y Gastronomía.

5. Crear un banco de semillas con diferentes accesiones de frijoles (CPA Aramís Pérez) en los años 2015 y 2016 para la selección de las semillas con mejores indicadores para su posterior propagación por los productores del territorio. En el banco de semillas sembrado en 2016 se realizó un encuentro con personal interesado durante la cosecha, para seleccionar las mejores accesiones. 
6. Ejecutar actividades de capacitación en las entidades productivas sobre temas prioritarios para la innovación agropecuaria local: producción de semillas, importancia y uso eficiente del agua, Medio ambiente, las buenas prácticas del PIAL y el Sistema de Innovación Agropecuaria Local (SIAL).

7. Realizar el evento regional Cadenas Agroalimentarias, Juventud y Género durante 4 años consecutivos como espacio de socialización de resultados y como un aspecto esencial en los ciclos de aprendizaje.

Los principales resultados de la aplicación de las acciones implementadas quedan expuestos en la siguiente tabla.

Tabla 3. Resultados de la implementación de la metodología del proyecto.

\begin{tabular}{ll}
\hline ETAPAS & RESULTADOS \\
\hline
\end{tabular}

ETAPAS

GIAL DE SEMILLAS

GIAL DE TABACALEROS

Diagnóstico Se realizó un pesquizaje para determinar cuáles productores se dedicaban a producir semillas para su consumo y otros destinos.

Establecimiento de la jerarquía de los objetivos

- Selección de productores de marcada experiencia en la actividad motivados y experimentados en estas prácticas.
- Definir las tecnologías que aplican en sus plantaciones, enfatizando en las más novedosas.

- Definir las especies a reproducir, enfatizando en las encaminadas a la sustitución de importaciones o que generen exportaciones.

- Establecer paquete tecnológico en la producción de semillas de cada especie.

- Se realizan ferias provinciales de intercambio de experiencias y de materiales de reproducción.

de las acciones derivadas del PIAL
- Se realizan acciones de capacitación sobre:

Manejo y conservación de suelos.

Uso racional del agua.

Empleo de abonos orgánicos y otras prácticas agroecológicas.
Se seleccionaron nueve productores de avanzada del territorio en el cultivo del tabaco, de conjunto con autoridades de la empresa TABACUBA.

- Selección de productores de marcada experiencia en la actividad y, a su vez, productores jóvenes menos experimentados, pero igualmente motivados.

- Definir las tecnologías que aplican en sus plantaciones, enfatizando en las más novedosas.

- Trabajar con variedades que mejor se adapten a las condiciones de las fincas.

- Mejorar el uso de buenas prácticas.

- Se realizaron encuentros de intercambio entre los productores.

- Se realizaron acciones de capacitación a través de las asambleas de las CCS donde se divulgaron las tecnologías a emplear en el cultivo del tabaco que mejor se adapten:

Conservación y mejora de 
suelos.

Uso racional del agua.

Empleo de abonos orgánicos y otras prácticas agroecológicas.

Medición del impacto

Evaluación de efectos

Diagnóstico

Como antecedentes en el patio se inició con

Establecimiento de la jerarquía de los objetivos especies de Begonias y Orquídeas.

- Se logra la producción de semillas de excelente calidad de las siguientes especies: col, frijoles (negro, colorado y blanco), maíz, ajíes, pepino, tomate.

- Se priorizó la producción de semillas de variedades autóctonas, mejor adaptadas a las condiciones locales.

\begin{abstract}
- Se incrementaron los conocimientos sobre temas tales como:
\end{abstract}

Conservación y mejora de suelos.

Uso racional del agua.

Empleo de abonos orgánicos y otras prácticas agroecológicas.

- Se incrementa la producción de semillas de las especies seleccionadas (prioridad a las locales).

- Se precisa el logro de un valor de la producción de frijol de \$1 422300 en el período.

- Caracterizar las variedades de plantas ornamentales que dispone la productora en la fase de inicio.

- Definir la meta prevista para el incremento de nuevas variedades.

- Identificar las variedades de plantas ornamentales para su adquisición en la
- Se logró el incrementar el área plantada de la nueva variedad de tabaco negro SS-2006.

- Se incorporó un productor joven en la tecnología de tabaco tapado.

- Se profundizó en los conocimientos sobre los temas:

Cambio climático.

\section{Enfrentamiento a sequías prolongadas.}

- La utilización de la nueva variedad de tabaco negro SS-2006 resultó beneficiosa a la agricultura tabacalera en general, pues los rendimientos en la actual campaña tabacalera han resultado superiores a los de la etapa precedente.

- El incremento de los cultivos de tabaco tapado mejoran la economía de los cultivadores y del territorio en general.

- Se precisa el logro de un valor de la producción de tabaco de \$14 294327 en el período.

Como antecedente, los autores contaban sólo con una cría de codornices.

- Realizar tres intercambios y monitoreos con el ECM en la CPA Cuba Nueva para adquirir experiencias en la actividad de mini industria.

- Dos sesiones de capacitación sobre acceso a la comercialización 
localidad u otros territorios.

- Aplicar las prácticas fitotécnicas correspondientes a cada variedad para la sostenibilidad en su producción.

- Evaluar los resultados del patio de plantas ornamentales. (Álvarez, 2008)

Implementación de las acciones derivadas del

PIAL
- Se implementaron las acciones 1,3 y 7 definidas en el cuerpo del trabajo. con expertos en el tema.

- Capacitación sobre la obtención de licencia sanitaria.

- Visitas a cinco mini industrias con resultados reconocidos.

- Crear las condiciones necesarias en el hogar (el PIAL brindó apoyo) para comenzar la elaboración de los encurtidos.

- Iniciar el encurtido de huevos de codorniz.

- Iniciar el encurtido de vegetales y algunos dulces en conserva que hoy son deficitarios para su comercialización.

- Se logró elaborar diferentes encurtidos (huevos de codorniz, vegetales de buena calidad).

- Se logró comenzar la comercialización vinculada a una CPA insertada en el PIAL, de las producciones en mercados (ferias y otros).

Efecto 1. Se han obtenido diferentes variedades de encurtidos con buena calidad y aceptación por los consumidores. Se logró: estrategia agropecuaria local, la producción y la futura comercialización de plantas ornamentales.

- Incorporar otra opción para la conservación sana de alimentos.

Efecto 2. Los Grupos de Innovación Agropecuaria Local (GIAL) se consolidan como espacios de participación local en el municipio.

- Los indicadores de efecto: se logró la inserción del patio en el GIAL de plantas ornamentales.

- Como fuentes de datos y mecanismos de verificación: evaluaciones, muestreos, entrevistas/ encuestas y estudios de caso.
- Incorporar nuevos productos para la dieta de la población a precios accesibles.

Efecto 2. Incorporación al GIAL de la mini industria de un nuevo productor y su familia. Resultado de los indicadores de efecto:

- Incorporar a los integrantes de la familia al GIAL (2 masculinos y 2 
- Se diseminaron e incorporaron buenas prácticas de producción; diversas prácticas agroecológicas y especies adaptadas al cambio climático (cactus y otras especies suculentas).

- Incremento del número promedio de especies y variedades de plantas ornamentales y su propagación.

- Integración de las buenas prácticas del GIAL en los currículos docentes y líneas de investigación en las instituciones de enseñanza y científicas (CUM, MINED).

- Se logró un efecto económico de \$7 200.

Efecto 3. Mujeres de comunidades rurales participan y se benefician de los procesos de innovación.

- Se logró incorporar una mujer y equidad de género. femeninas, un joven y una joven), dando respuesta a la equidad de género y generacional.

- Obtener una fuente de ingresos a la economía familiar al cerrar el ciclo de producción de materia prima, comercialización del producto procesado y conservado en la mini industria.

- Se logró de un efecto económico de $\$ 14000$.

Efecto 3. Mujeres de comunidades rurales y urbanas participan y se benefician de los procesos de innovación.

- Se logró incorporar dos mujeres, equidad de género y la participación de la familia.

\section{Fuente: Elaboración propia.}

Durante del 2012 y hasta la actualidad su implementación ha contribuido al desarrollo local (Pomares, 2006) a partir de lo cual se han logrado los siguientes resultados:

1. La selección de tres accesiones de frijoles como las más destacadas para el territorio en este año 2016, una parte de las semillas se entregó a la UNISS y otra parte se encuentra en manos de un productor que se encargará de propagarlas a través del GIAL de semillas y observarlas en la siguiente etapa de reproducción.

2. La realización de actividades de capacitación en las Asambleas Generales de las CPA Aramís Pérez, CCS Sergio Soto y CCS Nieves Morejón sobre los temas identificación del PIAL, la producción del frijol y los problemas actuales y sobre el agua y su importancia para la vida. Se realizaron tres actividades más en saludo al 5 de junio, Día Mundial del Medio ambiente.

3. La realización de visitas de asesoría a las principales muestras de confianza y entidades participantes para el intercambio, encaminados a promover la divulgación de experiencias sobre las mejores prácticas y resultados que se han obtenido en los renglones productivos de interés para los mismos.

4. Se han realizado 4 Talleres en el CUM Silverio Blanco Núñez del municipio Cabaiguán (2013, 2015, 2016 y 2017) sobre Cadenas agroalimentarias y Juventud y género, con resultados satisfactorios y buena participación de las entidades del territorio. Se ha logrado que los productores intercambien con otros no solo del municipio sino de la provincia y la nación, mostrando niveles de comunicación satisfactorios.

5. La realización en el CUM de Cabaiguán de un encuentro expositivo y de chequeo al PIAL, a nivel provincial, en marzo de 2016 por parte de auditores salvadoreños, con excelentes resultados y la participación de las entidades y productores involucrados. 
6. La sensibilización y la divulgación de conocimientos en las entidades y productores sobre el empleo de prácticas de producción más amigables al Medio ambiente y su aplicación, también en la participación de jóvenes y mujeres en el quehacer económico de la localidad, según lo expresado por las autoridades de las mismas. Un ejemplo lo constituye el incremento de la aplicación de las prácticas agroecológicas en la CPA Aramís Pérez para el manejo de los cultivos con el consiguiente ahorro de portadores energéticos y beneficios al Medio ambiente (laboreo mínimo, empleo de estiércol y otras).

7. La motivación y creación de una actitud innovadora para la implementación de tecnologías en las fincas que mejoren los resultados productivos; la incorporación de mujeres y jóvenes en las diferentes labores contribuyendo a la mejora económica de la familia.

Se han logrado beneficios económicos, sociales y ambientales de la innovación a partir de la capacitación y el aprendizaje y la implementación progresiva de las buenas prácticas aportadas por el sistema de innovación local como parte de la estrategia municipal. Es significativa la creación de los GIAL y la plataforma local para la innovación agropecuaria, como espacios de participación para el fomento de la innovación sobre la base de procesos y dinámicas participativas donde los jóvenes y las mujeres de comunidades rurales participen y se beneficien prioritariamente.

Los beneficios económicos se estiman en un valor de $\$ 202.5$ por la capacitación de 721 participantes en 27 actividades, con un costo de 7.50 \$/hora por cada capacitación. Se aportaron \$15 737827 por los GIAL (tabla 3) y \$1 300 por la asesoría realizada a las cooperativas del municipio: CPA Juan González, CCS Nieves Morejón y CPA Nueva Cuba, como extensión del proyecto.

Se aportan nuevas fuentes de empleo, la incorporación de jóvenes, de la mujer rural y de zonas urbanas en diversas tareas productivas y en el hogar como ente activo en la producción de alimentos; además en las zonas urbanas en la conservación y diversificación de especies de plantas ornamentales.

\section{CONCLUSIONES}

Las acciones aplicadas contribuyeron a la motivación de los productores e incrementaron el nivel de conocimientos a partir de la capacitación e intercambio como contribución a la seguridad alimentaria.

Se implicaron los pobladores del lugar donde se ejecutaron las acciones, las cuales constituyeron una oportunidad para el desarrollo comunitario sobre la base de la divulgación y la extensión de las buenas prácticas y las innovaciones locales, con principios agropecuarios ecológicamente sostenibles.

\section{REFERENCIAS BIBLIOGRÁFICAS}

Álvarez, A. (2008). Plantas ornamentales en Cuba: usos, diversidad y amenazas. Revista del Jardín Botánico Nacional, 29, 83-100. http://www.uh.cu/centros/jbn/descargas/rjbn/xxix/rev7.pdf

De Armas N., Lorences, J. y Perdomo M. (2003). Aproximación al estudio de la metodología como resultado científico [no publicado]. Centro de Estudios de Ciencias Pedagógicas del Instituto Superior Pedagógico Félix Varela de Villa Clara.

Hernández, C. A., Carrasco, M., Garcés, R. y Casas, R. (2016). Colaboración y alianzas del Centro Universitario Municipal (CUM) en la Red Local de Conocimiento e Innovación: Estudio de caso de Camajuaní, Villa Clara, Cuba. 
Instituto Nacional de Ciencias Agrícolas. (2012). Diseminación del Fitomejoramiento Participativo en Cuba. Proyecto para Fortalecer la Innovación Agropecuaria Local (PIAL). III Fase. https://pial.ihatuey.cu/

Marzin, J., López, T. y Cid, G. (2003). Manual de capacitación para la implementación de la metodología del proyecto de acompañamiento al sistema de extensión agraria. Ministerio de la Agricultura y Centro de Investigaciones Agronómicas para el Desarrollo.

Pomares, H. (2006). Tecnologías para el desarrollo local. Un camino de propuestas e interrogantes. En Guzón, A. (Coord.), Desarrollo local en Cuba: Retos y perspectivas (pp. 107-130). Editorial Academia.

Ramírez N., Hernández A. y Marrero A. (2009). Las nuevas tecnologías de la información y el desarrollo rural: propuesta para un debate [Ponencia]. II Simposio Internacional de Extensionismo, transferencias de tecnologías, aspectos socioeconómicos y desarrollo agrario sostenible de la Estación Experimental Indio Hatuey, Matanzas, Cuba.

Romero, M. I., Alejandro, M. y Delgado, S. (2009). Género, Participación y Poder. Manual técnicopráctico de la ANAP. Editorial Caminos.

Sánchez de Puerta, F. (1996). Extensión agraria y desarrollo rural: Sobre la evolución de las teorías y praxis extensionistas. Editorial Ministerio de Agricultura, Pesca y Alimentación. https://www.mapa.gob.es/ministerio/pags/biblioteca/fondo/pdf/8233 all.pdf

Tejeda, G. (2015). El enfoque de cadena como contribución a la sostenibilidad alimentaria. Proyecto: "Producción y conservación de semillas en la agricultura urbana y suburbana como componente esencial de la soberanía alimentaria». Editorial PROAGRU. 